Andrés Azuero Rodríguez

andres.azuero@correounivalle.edu.co

Calle 4B Nro 36-00

Cali-Colombia

Magister en Administración de la Universidad del Valle.

Docente del Departamento de Administración y Organizaciones de la Facultad de Ciencias de la Administración de la Universidad del Valle. Miembro del Grupo de Investigación Humanismo y Gestión (Categoría A1 Colciencias 2010). Docente de la Escuela de Responsabilidad Social del Valle del Cauca ERSEV.

Jenny Marcela Melo jmarcela.melo@gmail.com Calle 4B Nro 36-00 Cali-Colombia

Administradora de Empresas, Maestranda en Ciencias Sociales

(UNLP, Argentina), miembro del Grupo de Investigación Humanismo y Gestión de la Facultad de Ciencias de la Administración de la Universidad del Valle, Categoría A1 Colciencias.

Mónica García monica.garcia@correounivalle.edu.co Calle 4B Nro 36-00 Cali-Colombia

Ingeniera Industrial, Magister en Administración de Empresas de la Universidad del Valle. Master of Management, Universidad de Tulane, New Orleans. Coordinadora de la línea de investigación en Gestión Humana del Grupo de Investigación Humanismo y Gestión, Categoría A1 Colciencias.

Artículo Tipo: de reflexión Según Clasificación Colciencias

Fecha de recepción: 10 de Febrero de 2011 Fecha de corrección: 22 de junio de 2011 Fecha de aprobación: 22 de junio de 2011

\section{Una presentación de la región socialmente responsable del Norte del Cauca}

\author{
A presentation of the socially responsible region of \\ Northern of Cauca \\ Une présentation de la région socialement \\ responsable du Nord du Cauca
}

\section{Resumen}

Las Regiones Socialmente Responsables como estrategia territorial de la Responsabilidad Social Empresarial (RSE) están siendo desarrolladas en distintos lugares de Colombia. En el caso particular del Norte del Cauca, la Región Socialmente Responsable surge como una iniciativa de los empresarios con el ánimo de dar respuesta a las necesidades locales y apostar a la construcción de un proceso de intervención social en el marco de una agenda regional de desarrollo, que incluya la perspectiva de múltiples actores como empresarios de las zonas francas asentadas en la zona, gobiernos locales, academia y representantes de la comunidad.

Este artículo de reflexión se propone describir el proceso de construcción colectiva de la Región Socialmente Responsable del Norte del Cauca. Se presenta en tres partes: en la primera se describen los principales referentes conceptuales atinentes a la Responsabilidad Social Empresarial y la Región Socialmente Responsable. En la segunda parte se exponen las principales características de la zona del norte del Cauca como el contexto de aplicación de la estrategia. En la última parte se presenta la estrategia Región Socialmente Responsable en el Norte del Cauca, los hitos del proceso de articulación de actores que ha permitido la formación de la estrategia y se presentan unas reflexiones y conclusiones preliminares sobre los aprendizajes logrados a esta altura del proceso.

Palabras clave: región socialmente responsable, Norte del Cauca, responsabilidad social empresarial, Colombia. 


\section{A presentation of the socially responsible region of Northern of Cauca}

Una presentación de la región socialmente responsable del Norte del Cauca

Une présentation de région socialement responsable du Nord du Cauca

\section{Une présentation de région socialement responsable du Nord du Cauca}

Una presentación de la región socialmente responsable del Norte del Cauca

A presentation of the socially responsible region of Northern of Cauca

\section{Abstract}

Socially Responsible Territories as spatial strategy of Corporate Social Responsibility (CSR) are being developed in different parts of Colombia. In the case of Northern Cauca Region emerges as an initiative of entrepreneurs with the aim of responding to local needs and bet on the construction of a process of social intervention in the framework of a regional development agenda, including the prospect of multiple actors and entrepreneurs settled zones in the area, local governments, academia and representatives of the community.

This reflection article aims to describe the process of collective construction of the Socially Responsible Territory of Northern Cauca. It comes in three parts: the first describes the main conceptual references about Corporate Social Responsibility and Socially Responsible Region. The second part outlines the main features of the area of northern Cauca and the context of implementing the strategy. In the last part presents the strategy Socially Responsible Region in Northern Cauca, the milestones of the process of coordination of actors that has allowed the formation of the strategy and present some preliminary thoughts and findings on the learning achieved at this stage of the process.

Keywords: socially responsible region, Norte del Cauca, corporate social responsibility, Colombia.

\section{Résumée}

Les Régions Socialement Responsables comme stratégie territoriale de la Responsabilité Sociale des Entreprises (RSE) se développent sur plusieurs endroits de la Colombie. Pour le cas particulier du Nord du Cauca, la Région Socialement Responsable surgit comme une initiative des entrepreneurs pour donner une réponse aux besoins locaux et pour parier la construction d'un processus d'intervention sociale dans le cadre des politiques régionales de développement, qui puisse inclure la perspective de multiples acteurs : des entrepreneurs des zones franches situés sur la zone, des gouvernements locaux, l'académie et des représentants de la communauté.

Cet article de réflexion essaie de décrire le processus de construction collective de la Région Socialement Responsable du Nord du Cauca. Il se présente sous trois parties : dans la première partie on décrit les principaux référents conceptuels de la Responsabilité Sociale des Entreprises et de la Région Socialement Responsable. La deuxième partie présente les principales caractéristiques de la zone du Nord du Cauca à façon de contexte de l'application de la stratégie. Sur la dernière partie on trouve la stratégie Région Socialement Responsable dans le Nord du Cauca, les moments significatifs du processus d'articulation des acteurs qui a permis la formation de la stratégie, et se présentent aussi des réflexions et des conclusions préliminaires sur les apprentissages atteints sur ce point du processus.

Mots clef: région socialement responsable, Norte del Cauca, responsabilité sociale des entreprises, Colombie. 


\section{Una presentación de la región socialmente responsable del Norte del Cauca}

\section{Introducción}

LaS características y los impactos de las estrategias de Responsabilidad Social Empresarial cobran cada vez más importancia en los temas relacionados con el desarrollo regional. Ante los diversos retos derivados de los indicadores económicos, sociales y ambientales en las regiones del país se evidencia la necesidad de construir procesos de intervención social en los que participen todos los actores del desarrollo para consolidar una visión compartida de futuro. En ese sentido, una iniciativa en la que se puede canalizar este proceso es el programa "Región Socialmente Responsable". En el suroccidente colombiano, el caso más destacado de aplicación se ubica en la región del Norte del Cauca, que es una región que posee indicadores de crecimiento económico positivo, pero que aún enfrenta situaciones problemáticas en materias social y ambiental. Ello ha dado como resultado la necesidad de establecer una agenda regional de desarrollo en la que se ha utilizado como referente la Responsabilidad Social Empresarial. La revisión de este caso es importante para evidenciar las características de un proceso de alcance regional. Por lo anterior, el propósito de este documento es: 1) Describir algunos referentes teóricos de la Responsabilidad Social Empresarial y la Región Socialmente Responsable; 2) Describir las características de la zona del Norte del Cauca como contexto propicio para el desarrollo de estrategias de responsabilidad social empresarial; 3) Describir la estrategia Región Socialmente Responsable en el Norte del Cauca. 4) Presentar algunas reflexiones y conclusiones sobre el avance del proceso.

\section{La responsabilidad social empresarial: un eje articulador}

El concepto de Responsabilidad Social Empresarial es el pilar fundamental sobre el cual el
Programa Región Responsable tiene sentido. Como eje transversal del proceso de revisión del plan se avanzó en una profundización conceptual que permitiera comprender el significado, y establecer las características y matices de este concepto. A continuación se presentan los rasgos principales de la revisión que se adelantó y que permitió construir una comprensión al respecto de esta práctica empresarial

La RSE es una noción que en la última década ha logrado instalarse en el contexto de las organizaciones empresariales del mundo entero. Si bien hoy se encuentran numerosas y variadas definiciones provenientes de distintos actores, en su mayoría de organizaciones empresariales, ONG's y Organismos Internacionales, no es una construcción reciente, deviene en un proceso que encuentra sus primeros aportes en los años 50 (Carroll, 1999) ${ }^{2}$. Desde los lejanos años 50 hasta los actuales los debates en torno al papel que puede jugar la empresa con respecto al desarrollo social y a los desafíos medioambientales han avanzado de forma significativa. Garriga y Melé (2004) realizaron un mapeo de las principales teorías y enfoques sobre la RSE y las clasificaron en cuatro grupos de teorías: instrumentales, políticas, integradoras y éticas.

En las teorías instrumentales la empresa es vista principalmente como un vehículo para la creación de riqueza y sus actividades sociales como un medio para alcanzar resultados económicos. Este grupo incluye tres tipos de teorías: en primer lugar las que consideran que el criterio supremo para evaluar las actividades sociales empresariales es la maximización del valor para el accionista, en la cual se enmarca la mirada ya clásica de Friedman (1970). En segundo lugar se encuentran las teorías centradas en destinar recursos para lograr objetivos sociales en el largo plazo y crear ventaja competitiva. En estas teorías

1. La Región Socialmente Responsable es un Programa puesto en marcha por la Oficina del Global Compact en Colombia, que tiene como objeto complementar el desarrollo local promovido por el PNUD el cual genera procesos en diferentes unidades geográficas. La intención es que a través de esas unidades, crear condiciones sociales, políticas y productivas que promuevan el desarrollo integral de la región, entendida como un espacio geográfico donde las personas comparten las mismas características culturales, sociales, políticas y de producción, que son la unidad básica para el desarrollo de la región.

2. En 1953 Howard Bowen (citado en Carroll 1999) proponía que las responsabilidades sociales del hombre de negocios se referían a "las obligaciones de seguir aquellas políticas, tomar decisiones, o seguir esas líneas de acción que fuesen deseables en términos de objetivos y valores de la sociedad". 
se incluyen tres enfoques: a) inversiones sociales en contextos competitivos, propuesta desarrollada por Porter y Kramer (2002), b) estrategias basadas en una visión de recursos naturales y capacidades de la empresa y c) estrategias para la base de la pirámide, esto es, estrategias para aumentar el consumo de la población pobre (Prahalad y Hart, 2002)3. El tercer tipo de teorías se relaciona con el mercadeo de causa-social, cuyo objetivo principal es aumentar las ventas e ingresos de la empresa 0 la relación con los clientes creando una imagen de marca mediante la adquisición o la asociación con la dimensión ética o la dimensión de responsabilidad social.

Las teorías políticas se centran en las interacciones y conexiones entre la empresa y la sociedad, sobre el poder y posición de las empresas y su responsabilidad inherente, e incluyen consideraciones y análisis políticos en el debate sobre RSE. En la variedad de enfoques políticos, Garriga y Melé distinguen tres corrientes: el constitucionalismo corporativo, la teoría del contrato social integrador y la ciudadanía corporativa La primera se basa en las propuestas de Davis (1960) sobre el rol del poder que las empresas tienen en la sociedad y el impacto social de ese poder. La segunda es la teoría del contrato social integrador, propuesta por Donaldson y Dunfee $(1994,1999$, citado por Garriga y Melé, 2004), considera que el contrato social implícito que existe entre la empresa y la sociedad supone algunas obligaciones indirectas de la empresa hacia la sociedad, de las cuales se deriva la RSE. Con respecto a la ciudadanía corporativa, el BID (2007), retomando a Garriga y Melé (2004) indica que éste es un concepto que contiene tres enfoques diferentes: una visión limitada, que comprende la filantropía corporativa, la inversión social y el asumir ciertas responsabilidades con respecto a la comunidad; una visión equivalente a la RSE; aunque esta última tiene otra interpretación más amplia, según la cual las empresas empiezan a tratar del tema de la ciudadanía cuando el gobierno falla en la protección de la ciudadanía incluso en el ámbito global. A pesar de las diferencias existentes en este grupo de teorías, se puede identificar algunos puntos de convergencia: un fuerte sentido de responsabilidad de la empresa con respecto a las comunidades, la consideración de formar parte de asociaciones para fomentar el desarrollo de las comunidades y la creciente preocupación por el medio ambiente.
En las teorías integradoras la empresa está enfocada en la satisfacción de las demandas sociales. El argumento de esta orientación es que las empresas dependen de la sociedad para su existencia, continuidad y crecimiento. Este grupo comprende el enfoque de la gestión de los asuntos sociales ( Wartick and Mahon, 1994), el principio de responsabilidad pública, (Preston and Post, 1975;1981), el enfoque de gestión de los stakeholders, y el desempeño social corporativo. El primero se orienta a los procesos de inclusión de las demandas sociales y el proceso de toma de decisiones basado en estas exigencias. El segundo sostiene que un comportamiento empresarial adecuado deriva de una política pública, la cual debe comprender leyes, reglamentos, aspectos de opinión pública, la emergencia de temas sociales y las prácticas de implementación del marco legal. El enfoque de gestión de los stakeholders se orienta hacia las personas o grupos que afectan o se ven afectados por las políticas y prácticas empresariales; en este enfoque se analiza la cooperación entre los stakeholders y la empresa para maximizar los beneficios mutuos y la eficiencia de las estrategias de manejo de intereses conflictivos. El desempeño social corporativo incorpora la búsqueda de la legitimidad social por medio de procesos que ofrezcan respuestas adecuadas a determinados problemas sociales.

Finalmente, las teorías éticas se basan en los requerimientos éticos que tejen la relación entre la empresa y la sociedad. Los enfoques que pueden distinguirse en este grupo son: la teoría normativa de stakeholders, los derechos universales, el desarrollo sostenible y el enfoque del bien común. La teoría normativa de stakeholders examina las obligaciones fiduciarias de la empresa hacia todos sus grupos de interés: proveedores, clientes, empleados, accionistas y comunidad. Su puesta en práctica exige tomar como referencia alguna teoría ética. El desarrollo sostenible indica que, en consideración de las generaciones futuras, la empresa debe buscar una triple cuenta de resultados (en inglés triple bottom line) que incluye aspectos económicos, sociales y medioambientales. El enfoque del bien común sostiene que la empresa debe contribuir al bien común porque es parte de la sociedad. La empresa cuenta con muchos medios para conseguirlo: creando riqueza y suministrando bienes y servicios de una manera justa y eficiente

3. Resulta interesante notar que las estrategias con la base de la pirámide están ganando mucha importancia en el contexto empresarial y académico, llegando a conformar un acervo documental propio. Para una ilustración al respecto SEKN (2010), Márquez, P; Reficco, E. y Berger, G. (2009), y Gardetti, M.A y Muro, A.T. (.2009). 


\begin{tabular}{|c|c|c|c|}
\hline Inactivo & Reactivo & Activo & Pro/interactivo \\
\hline $\begin{array}{l}\text { Autorresponsabilidad } \\
\text { corporativa } \\
\text { Hacia adentro } \\
\text { "Haciendo las cosas bien" } \\
\text { "Me está yendo bien" } \\
\text { "Sólo hazlo" }\end{array}$ & $\begin{array}{c}\text { Sensibilidad social } \\
\text { corporativa } \\
\text { De afuera hacia adentro } \\
\text { "No hagas las cosas mal" } \\
\text { "Yéndome bien y haciendo } \\
\text { el bien" } \\
\text { "Sólo no lo hagas" }\end{array}$ & $\begin{array}{c}\text { Responsabilidad social } \\
\text { corporativa } \\
\text { De adentro hacia afuera } \\
\text { "Haciendo las cosas } \\
\text { correctas" } \\
\text { "Haciendo el bien" } \\
\text { "Haz lo justo" }\end{array}$ & $\begin{array}{c}\text { Responsabilidad corpora- } \\
\text { tiva de lo social } \\
\text { De adentro / afuera - Hacia } \\
\text { / adentro-afuera } \\
\text { “Haciendo bien las cosas } \\
\text { correctas" } \\
\text { "Yéndome bien por hacer e } \\
\text { bien” } \\
\text { “Sólo haz lo justo" }\end{array}$ \\
\hline \multicolumn{2}{|c|}{ Eficiencia } & Equidad / Ética & Eficacia \\
\hline $\begin{array}{l}\text { Motivo utilitarista: } \\
\text { maximización de la utilidad }\end{array}$ & $\begin{array}{l}\text { Enfoque de los deberes } \\
\text { negativos: ganancias } \\
\text { trimestrales y capitalización } \\
\text { en los mercados }\end{array}$ & $\begin{array}{c}\text { "Deber positivo" o “basado } \\
\text { en la virtud" : valores } \\
\text { (rentabilidad del largo } \\
\text { plazo) }\end{array}$ & $\begin{array}{c}\text { Enfoque del deber } \\
\text { interactivo: rentabilidad de } \\
\text { mediano plazo y sostenibi- } \\
\text { lidad }\end{array}$ \\
\hline Indiferencia & Conformidad & Integridad & Discurso Ético \\
\hline \multicolumn{2}{|c|}{ Gestión de la empresa y la sociedad } & $\begin{array}{c}\text { Gestión de la empresa en la } \\
\text { sociedad }\end{array}$ & Gestión empresa - sociedac \\
\hline \multicolumn{2}{|c|}{ Confía en mi } & Pruébamelo & $\begin{array}{c}\text { Involúcrate / Comprométete } \\
\text { conmigo; Únete a mí }\end{array}$ \\
\hline
\end{tabular}

Fuente: basado en Van Tulder y Van der Zwart (2006).

$y$, al mismo tiempo, respetando la dignidad y los derechos fundamentales de los individuos.

Van Tulder y Van der Zwart (2006) ofrecen otra forma de analizar el nutrido acervo bibliográfico con que cuenta la RSE. Proponen realizar una clasificación a partir de cuatro enfoques: inactivo, reactivo, activo, y pro/interactivo, los cuales emergen de diferentes estados de desarrollo social y no son ni mutuamente excluyentes, ni representarían los mejores modelos. En la tabla 1 se presenta una síntesis con los principales rasgos de cada enfoque.

El enfoque inactivo refleja la noción clásica de Friedman (1970). Los empresarios están particularmente interesados en "hacer las cosas bien"; no se plantean preguntas éticas o sobre los fundamentos de lo que hacen. La RSE presente en este enfoque puede denominarse como "autoresponsabilidad empresarial" y está encaminada a la maximización de las ventas y los beneficios.

En el enfoque reactivo la motivación de RSE es principalmente basada en "deberes negativos", donde la empresa está forzada a conformarse con las normas definidas por los stakeholders sobre comportamiento apropiado. Ambos enfoques, inactivo y reactivo, se enfocan en gran medida en indicadores de salida como retornos y productivi- dad y están fuertemente orientados a la riqueza y a los recursos. Las relaciones con la sociedad y la comunidad de stakeholders son relativamente involuntarias.

Un enfoque activo de la RSE representa una orientación empresarial más ética. Los empresarios que asumen este enfoque están explícitamente inspirados en valores éticos y virtudes (o deberes positivos), sobre los cuales formulan los objetivos de la compañía. Finalmente, el enfoque proactivo / interactivo se basa en un discurso ético y en una fuerte relación con los stakeholders. Se orienta hacia la eficacia, buscando rentabilidad y sostenibilidad, emprendiendo acciones justas, correctas y éticas.

Este mapa conceptual que ofrecen Garriga y Melé permitió tener una mirada amplia de los distintos matices y orientaciones que puede tener la RSE4. Considerando que los grupos de estas teorías son más bien "tipos ideales" y no se encuentran en la realidad en un "estado puro", se propone un entendimiento de la RSE que dé soporte a la estrategia de Region Responsable en los siguientes términos: la responsabilidad social empresarial es un enfoque de gestión de empresa que se expresa en acciones concretas en todas las áreas funcionales de una organización. Para el caso colombiano es necesario entender que la RSE 
se instala en una larga experiencia filantrópica de las empresas. Y que la RSE viene a ser un estado posterior de evolución de esta experiencia (Gutiérrez, Avella y Villar, 2004).

Este enfoque de gestión implica tanto un compromiso voluntario de incorporar valores éticos en la toma de decisiones empresariales como una nueva forma de relacionarse con los grupos de interés / stakeholders. En este sentido, la RSE implica una transformación de la relación tradicional entre empresa y sociedad; en esta nueva perspectiva la empresa no ignora al entorno donde opera, se orienta al logro de un desarrollo integral partiendo de la consideración de su esfera de influencia ${ }^{5}$, que permite entender los campos de acción y los diferentes alcances de la RSE, particularmente cuando se piensa en la construcción de estrategias locales $y$ regionales.

La RSE debe trascender el mero cumplimiento del marco normativo, y comprometerse con las dimensiones sociales, ambientales y económicas en las que la empresa tiene una relación y un impacto. Un elemento fundamental es que el tipo de acciones que una empresa adelante en el marco de su responsabilidad social empresarial se relacione directamente con el sector del cual hace parte y de las particularidades de su contexto inmediato.

\subsection{La Región Socialmente Responsable: Algunos referentes}

El replanteamiento de la relación empresa y sociedad implica la consideración de la actuación de la empresa en una dimensión territorial, vista desde nociones como esfera de influencia, cooperación territorial, ciudadanía responsable, entre otras, tanto por los impactos como por la capacidad de articularse con otros actores en torno a iniciativas de desarrollo local en una área geográfica determinada.

Al respecto de la exploración de la dimensión territorial de la RSE se pueden encontrar diversas iniciativas. En Europa, por ejemplo, se encuentra la Red Europea de Territorios Responsables (REVES) que define los territorios socialmente responsables como "un sistema territorial que conjuga el equilibrio entre los aspectos económicos, sociales, ambientales y culturales del modo de vida local buscando conseguir una mejor calidad de vida para los habitantes locales y otros agentes implicados, a través de un enfoque de gobernanza participativa" (Castillo, 2007:3).

De acuerdo con lo expuesto en el Segundo Encuentro Internacional de Regiones Responsables, realizado en 2009 en Barranquilla (Colombia) hay diferentes experiencias destacadas. En España, por ejemplo se encuentran los trabajos de Xavier Agulló (2009), sobre cómo consolidar el Puerto de Barcelona como un territorio socialmente responsable. En América Latina, se encuentra el trabajo de Gabriel Boero en la municipalidad de San Martin en el que se promueve el desarrollo del territorio a partir del trabajo articulado de diversos actores locales.

En Colombia, la perspectiva territorial de la Responsablidad Social Empresarial ha sido abordada desde el programa Región Socialmente Responsable. De acuerdo con Salinas (2010) el programa de Regiones Responsables fue creado en Colombia en 2007 como una iniciativa de la Red del Global Compact ${ }^{6}$ con el ánimo de ofrecer meca-

5. La esfera de influencia es un concepto desarrollado como una herramienta por el Foro Internacional de Líderes Empresarial (IBLF por sus siglas en inglés), que avanza en categorizar las responsabilidades que tiene una empresa. De acuerdo con el IBFL, las esferas de influencia tienen cuatro áreas: 1) Dentro del negocio principal, donde la compañía tiene mayor control directo y puede poner a funcionar los procedimientos gerenciales y administrativos; 2) Entre la compañía y sus socios de negocio, donde el nivel de control directo varía dependiendo de la naturaleza de la relación, pero, como mínimo, puede ejercerse una influencia fuerte para asegurarse que no ocurran violaciones a los derechos humanos. Cualquier documentación de la relación de negocios debería específicamente referirse a los estándares esperados de desempeño en los negocios; 3) Entre la compañía y la comunidad en la cual opera, donde el grado de influencia y control depende de un conjunto de factores sociales, económicos y medioambientales, y 4) Entre la compañía y los organismos de gobierno nacionales, donde probablemente la influencia.

6. Los Diez Principios del Global Compact están basados en Declaraciones y Convenciones Universales aplicadas en cuatro áreas: Derechos Humanos, Medio Ambiente, Estándares Laborales y Anticorrupción. Los principios son:1) Las Empresas deben apoyar y respetar la protección de los derechos humanos fundamentales reconocidos universalmente, dentro de su ámbito de influencia; 2) Las Empresas deben asegurarse de no ser cómplices de la vulneración de los derechos humanos; 3) Las empresas deben apoyar la libertad de Asociación y el reconocimiento efectivo del derecho a la negociación colectiva.; 4) Las Empresas deben apoyar la eliminación de toda forma de trabajo forzoso o realizado bajo coacción; 5) Las Empresas deben apoyar la erradicación del trabajo infantil; 6) Las Empresas deben apoyar la abolición de las prácticas de discriminación en el empleo y ocupación; 7) Las Empresas deberán mantener un enfoque preventivo que favorezca el medio ambiente; 8) Las empresas deben fomentar las iniciativas que promuevan una mayor responsabilidad ambiental; 9) Las Empresas deben favorecer el desarrollo y la difusión de las tecnologías respetuosas con el medio ambiente; 10) Las Empresas deben trabajar en contra de la corrupción en todas sus formas, incluidas la extorsión y el soborno. 
nismos prácticos colectivos para el ejercicio de la Responsabilidad Social. En la actualidad "Regiones Responsables" es un programa autónomo, con organización propia, con el apoyo del Pacto Global que se desarrolla en varias regiones de Colombia.

Una “Región Responsable” definida por el Global Compact es un territorio específico donde convergen las dinámicas de un sistema social particular, en el que los actores buscan desarrollar una alternativa de desarrollo desde sus propias capacidades y particularidades de su vida económica, social, política y cultural. En tanto región se entiende como un sistema interdependiente de relaciones colaborativas y de compromisos mutuos con una visión sostenible del territorio, que se garantiza a través de la construcción de redes de confianza y reciprocidad.

Este hecho implica un nuevo "Contrato social" que se enfoca, en principio, en la construcción y consolidación de una ciudadanía responsable que asuma sus responsabilidades, deberes y derechos; una nueva dinámica económica basada en la ética y la justicia social; la construcción de capacidades para el liderazgo social; y un ejercicio transparente, participativo y eficaz de la política en un modelo democrático de múltiples niveles de gobernabilidad del desarrollo (Salinas, 2010; citado en Betancourth, Azuero y Martinez: 2011:14).

El programa Región Socialmente Responsable, liderado por el Global Compact, propone la conformación de una coalición de empresarios, gobierno, y organizaciones sociales de la región, con tres objetivos básicos: lograr acuerdos y acciones coordinadas en favor del desarrollo local, por parte de todos los actores sociales, a través de múltiples escenarios de alianzas público-privadas; introducir el concepto de responsabilidad social empresarial como uno de los objetivos y como uno de los mecanismos para ayudar a lograr el desarrollo local; y establecer una agenda colectiva de responsabilidad social organizacional en marcha, basada en la implementación de los principios del Global Compact de las Naciones Unidas.

Según el pacto Global, de la configuración de este escenario de Región Responsable se pueden obtener beneficios de diversa índole; entre ellos: integración del plan de Responsabilidad Social de las empresas con la región; Articulación colectiva de la Responsabilidad Social; Coordinación de la Responsabilidad Social con la política pública; Escalamiento del impacto de las acciones de Responsabilidad Social; Creación de un entorno de desarrollo organizacional y empresarial positivo; y Formación de un ambiente de co-responsabilidad social, entre otros.

En Colombia, durante 2008, el Global Compact impulsó en siete regiones la puesta en marcha del modelo región socialmente responsable. Las regiones que se encuentran aplicando este modelo son: Norte del Cauca, Guabillo-Cundinamarca, Atlántico, Santander, Norte de Santander, el Altiplano Cundí boyacense, y Boyacá. En el marco de esta iniciativa, la Organización de las Naciones Unidas, junto a varias agencias de cooperación, organizaron y ofrecieron a empresarios del país, el Diplomado Empresas y Derechos Humanos.

\section{La experiencia de la Región Responsable en el Norte del Cauca}

\subsection{Una mirada al Norte del Cauca}

El 6 de junio de 1994 el departamento del Cauca $^{7}$ sufrió un fuerte sismo que generó una avalancha en el río Páez, ocasionando grandes pérdidas tanto de vidas como de infraestructura en los departamentos de Cauca y Huila. Esta tragedia afectó las oportunidades de crecimiento social y económico, profundizando las condiciones de pobreza de esta zona.

Como respuesta a esta situación, el Gobierno Nacional aprobó la Ley 218 de 1995, conocida como la Ley Páez, por la cual "se establecen exenciones tributarias para la zona afectada por la calamidad pública en los Departamentos del Huila y Cauca" ${ }^{8}$. Esta Ley incentivó la instalación de aglomeraciones empresariales bajo la figura de "parques industriales", los cuales se ubicaron principalmente en los municipios de Puerto Tejada, Santander de Quilichao, Caloto y Villa Rica. Ver en la gráfica 1 la división político administrativa del departamento del Cauca.

Gracias a la importación de bienes de capital libres de impuestos, que permitió esta

7. El Departamento de Cauca está situado en el suroeste de Colombia, entre las regiones andina y pacífica; cuenta con una superficie de $29.308 \mathrm{~km} 2$ lo que representa el $2.56 \%$ del territorio nacional. Limita por el Norte con el departamento del Valle del Cauca, por el Este con los departamentos de Tolima, Huila y Caquetá, por el Sur con Nariño y Putumayo y por el Oeste con el océano Pacífico.

8. En www.secretariasenado.gov.co/.../ley/1995/ley_0218_1995.html se puede consultar el documento completo de la Ley. 
Ley se generó una oportunidad para modernizar el aparato productivo de la economía caucana ${ }^{9}$.

Este nuevo asentamiento industrial, inexistente antes de la ley, impulsó el cambio de la crecido en participación y también se ha diversificado. Para lo corrido de la vigencia de la ley Páez el valor agregado industrial del Cauca ha crecido consistentemente. Hoy en el norte del Cauca, especialmente en jurisdicción de los municipios de Caloto, Guachené, Villarica y Puerto Tejada, hay asentadas 139 empresas en varios parques industriales que generan unos 20.000 empleos directos e indirectos (6.500 directos).

La creación de estos parques industriales ha traído un impacto positivo en la estructura econó-

\section{Gráfica 1. Mapa del Departamento del Cauca}

mica de la zona y continua consolidándose (Alonso, 2006), sin embargo, las expectativas impuestas en la generación de empleo local no han sido totalmente satisfechas. (Betancourt, Azuero y Martinez, 2010).

En el departamento del Cauca alrededor del $40 \%$ de la población es afrodescendiente y se ubica principalmente en la zona norte del departamento, representando en la zona casi un $90 \%$ de la población. Según el Plan de Desarrollo y la Agenda Regional del Cauca (AMUNORCA, 2006), la zona del Norte del Cauca presenta unos indicadores bastantes críticos con relación a la calidad de vida de los habitantes: el $39,7 \%$ de los hogares viven en condiciones de indigencia y el $77 \%$ en condiciones de pobreza; el $57,5 \%$ de los habitantes presenta más de una necesidad básica insatisfecha, solamente el $54,5 \%$ de

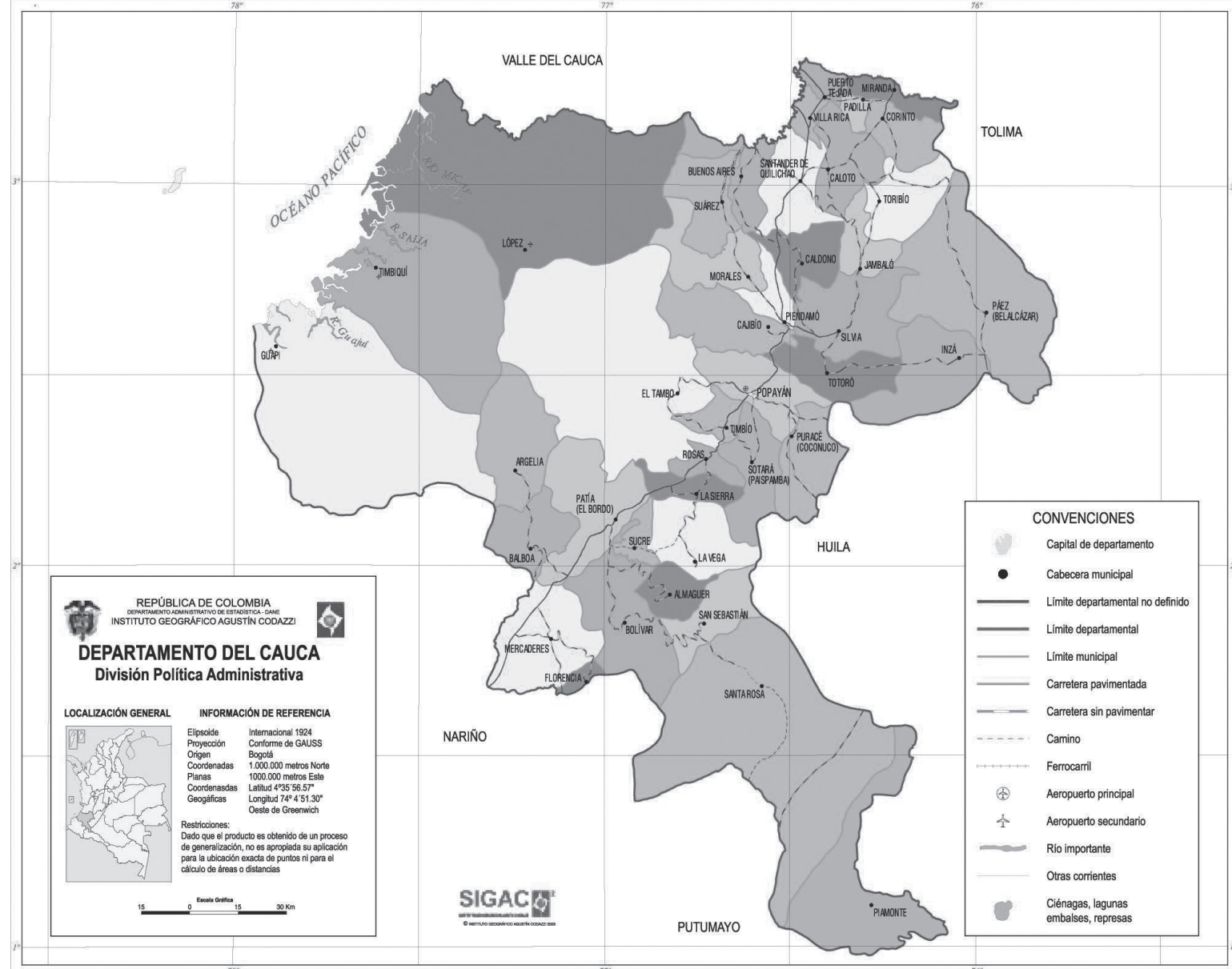

Fuente: IGAC (2009).

9. De acuerdo con la Ley 218 y con una modificación posterior del artículo 42 de la Ley 383 de 1997, la cobertura geográfica de las exenciones tributarias y otros beneficios comprende los municipios de: Caldono, Inzá, Jambaló, Toribío, Caloto, Totoró, Silvia, Páez, Santander de Quilichao, Popayán, Miranda, Morales, Padilla, Puracé, Tambo, Timbío , Cajibío, Piendamó, Sotará, Buenos Aires, La Sierra, Puerto Tejada, Corinto, Patía y Suárez (Alonso, 2006). 
los hogares cuenta con una vivienda digna y con posibilidad de acceder al sistema escolar.

Según un estudio de la Universidad ICESI sobre la ley Páez (Alonso y Lotero, 2008) existen en la zona serios problemas en términos de formación y de infraestructura. En la gráfica 2 se presenta una distribución de la población según el nivel educativo. La población se concentra en los niveles educativos con primaria incompleta, secundaria incompleta y secundaria completa. Debido a esta situación, las demandas por empleos con niveles altos de cualificación son provistos por trabajadores provenientes de municipios como Cali, aunque la mayor parte del empleo nuevo en niveles medios y bajos proviene de la región y sigue en aumento.

\section{Gráfica 2. Distribución de la población según el} nivel educativo

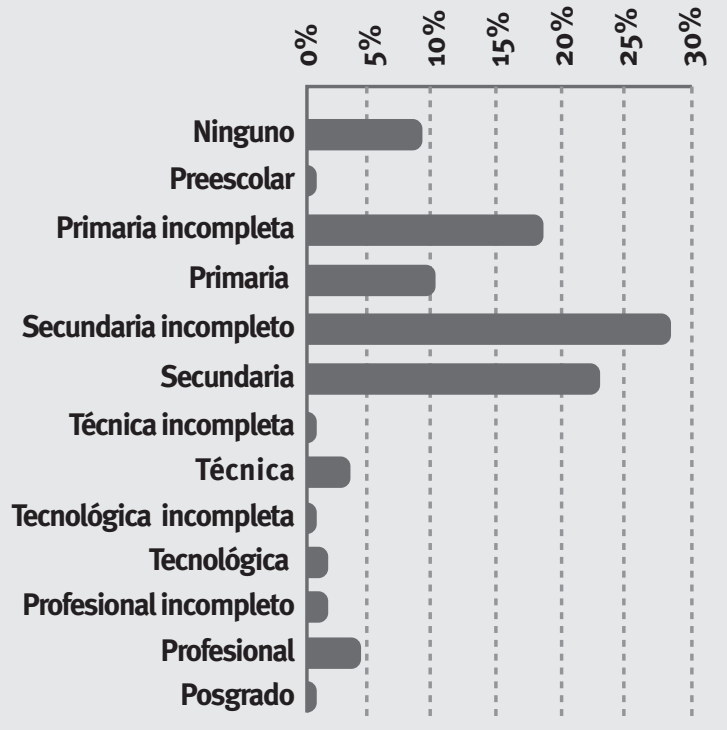

Fuente: Alonso y Otero (2008:39).

Las condiciones de infraestructura son limitadas: la infraestructura energética de la zona presenta inestabilidad en el servicio; el servicio de acueducto presenta problemas con la calidad del agua para consumo humano y animal, incluso, no es apto en muchas ocasiones para los procesos de producción; la red de servicios de salud es insuficiente para atender las necesidades de la zona; las telecomunicaciones y las vías de comunicación también tienen serias limitaciones.

Para asegurar la continuidad de las empresas en la zona del norte de Cauca, el Gobierno Nacional expidió el Decreto 780 de 2008 , en el cual se autorizó la creación de Zonas Francas en la zona, las cuales generan una perspectiva de permanencia de al menos 30 años más en el Norte del Cauca, con el compromiso de más inversión y generación de empleo local.

A continuación se exponen los que consideramos los principales hitos del proceso de construcción de la Estrategia Región Responsable. Estos han sido identificados a partir de un proceso de acompañamiento realizado por la Facultad de Ciencias de la Administración de la Universidad del Valle.

\subsection{La Región Socialmente Responsable en el Norte del Cauca}

Para el caso particular del Norte del Cauca, la estrategia Región Socialmente Responsable encuentra eco entre los empresarios asentados en la zona y se identifican elementos de las condiciones sociales de la región y los retos que ello imponen al accionar de las empresas. Una de las principales situaciones que se presentan en la zona es el problema de la pobreza. Estas condiciones que rodean a los habitantes de la zona, se combinan con el pasado histórico de esclavitud que les antecede y configura una región de diversidad cultural y con retos y desafíos propios.

Ante este panorama, como resultado del trabajo articulado de diversas instituciones y actores de la región, se elabora un proyecto de investigación financiado por Colciencias cuyo objetivo general es articular a los diversos actores características cuyo objetivo general es articular a los diversos actores públicos y privados de la región Norte del Cauca en una estrategia de Región Socialmente Responsable para lograr el desarrollo integral de las empresas, las instituciones, los municipios y las comunidades de los municipios de Caloto, Guachené, Puerto Tejada y Villarica.

Para esto, se han previsto cinco objetivos específicos relacionados con: caracterizar tanto la región donde operan las empresas del parque industrial del Norte del Cauca, como la base empresarial y las capacidades sociales instaladas; realizar un estudio de prospectiva regional para el Norte del Cauca; diseñar un plan de acción en Responsabilidad Social para la región Norte del Cauca, y realizar un proceso transversal de sensibilización a los actores de la base empresarial de la región en el proceso de Región Responsable.

Para el desarrollo de este proyecto de investigación aplicada se combinarán métodos cualitativos y cuantitativos. La estrategia de desarrollo del proyecto está concebida en cinco fases articuladas. 
En la Fase 1 (Caracterización de la región) se hará una lectura general de las condiciones del territorio como punto de partida, para identificar las características generales y las necesidades particulares de la región. Con esta información se construye una caracterización del contexto de operación de las empresas y las instituciones y sus grupos de interés en la región. Esta fase la coordina la Facultad de Ciencias de la Administración de la Universidad del Valle.

En la Fase 2 (Capacidades empresariales y capacidades sociales instaladas) se hará un análisis de la base empresarial de la región y se realizará una lectura del capital social y los procesos sociales en términos organizativos y participativos que puedan promover el desarrollo de la región. Esta fase la coordina la Cámara de Comercio del Cauca y la Corporación Vallenpaz.

En la Fase 3 (Escenarios de realidades y futuros) contando con los insumos de las dos fases anteriores, se realizará un estudio de prospectiva territorial para identificar los escenarios posibles de futuro de la región. Esta fase la coordina la Facultad de Ciencias de la Administración de la Universidad del Valle.

En la Fase 4 (Construcción de acuerdos) se promoverá la construcción de los pactos colectivos de acciones en Responsabilidad Social para toda la región, a través del diseño de un plan regional que permita la consolidación del entramado empresarial de la región y sus grupos de interés para propiciar escenarios de desarrollo local.

En la Fase 5 (Preparación transversal), transversal a todo el desarrollo del proyecto se realizará una sensibilización de los actores para involucrar a la base empresarial en el proceso de región responsable, y desarrollar capacidades empresariales y sociales en temas de responsabilidad social, derechos humanos y desarrollo.

Se ha previsto que al final del proceso de las cinco fases se cuente con un modelo de formadores en Responsabilidad Social; una comunidad docente crítica capacitada como formadores en la red de Formación en RSE, y actores de la zona sensibilizados tanto en RSE como en Derechos Humanos y Desarrollo.

Igualmente, como parte de los productos académicos se contará con los documentos de estudio de la caracterización regional, las capacidades empresariales y sociales, y prospectiva regional del Norte del Cauca, que sirven de línea base para futuros desarrollos en el tema.

La Región Socialmente Responsable Norte del Cauca es un camino aún en construcción. Las actividades desarrolladas en el marco de la iniciativa son el punto de partida para la consolidación de un proceso de largo aliento, donde sólo el trabajo colaborativo, constante y sostenido permitirá un cambio visible en el largo plazo.

\subsection{Hitos del proceso de construcción de la Estrategia de Región Responsable}

Ante los retos que imponen las condiciones socio -económicas de la región norte del Cauca, las expectativas de generación de empleo local derivadas de la llegada de los parques industriales, y la perspectiva de configuración de los parques en zonas francas, un grupo de empresarios e instituciones interesados por el desarrollo de la región (Pavco, Familia, Propal, Forsa, Fundesimpa, Cencauca, Papeles del Cauca Kimberly, Badelca, Vallenpaz, Cámara de Comercio del Cauca, Comfacauca y las alcaldías de Puerto Tejada, Guachené, Villarica y Caloto), inició un acercamiento con la Oficina del Global Compact en Colombia. Dado el enfoque de esta institución, que articula actores de diversos sectores en pro del desarrollo social, desde las empresas se organizó la visita de un consultor para iniciar un proceso de sensibilización en temas de Responsabilidad Social dirigido a los empresarios del Norte del Cauca.

A partir del interés generado en esta visita, desde el sector empresarial se promueve el desarrollo del Programa Región Socialmente Responsable en la zona. La primera actividad con que se inicia este programa fue el Diplomado Empresas y Derechos Humanos ${ }^{10}$, en el que participaron empresarios, alcaldes y académicos de la región. La metodología del diplomado incluyó la realización de un diagnóstico de Responsabilidad Social Empresarial para

10. El Diplomado fue organizado por la Red del Pacto Global en Colombia, junto a la Agencia de los Estados Unidos para el Desarrollo Internacional (USAID), la Agencia de Cooperación Técnica Alemana GTZ, el Alto Comisionado de las Naciones Unidas para los Refugiados (ACNUR) y la Oficina de Naciones Unidas contra las Drogas y el Delito (UNODC).con el propósito de "promover conciencia entre los empresarios sobre la necesidad de prevenir que sus actividades empresariales puedan producir efectos negativos sobre los derechos humanos, el desplazamiento forzado y la corrupción, o afectaciones a los derechos de las comunidades vecinas, sus trabajadores o al medio ambiente”. Más información en http://www.empresasyderechoshumanos.org 
cada institución participante y la articulación de un primer borrador de un plan regional de RSE. El propósito del plan regional es fomentar acciones colaborativas para concentrar las inversiones y esfuerzos individuales en torno a ciertas actividades focalizadas para alcanzar un mayor impacto en la región. De acuerdo a la información de los diagnósticos y al trabajo de un grupo de participantes se seleccionan como temas prioritarios para el primer año en la Región Responsable: saneamiento básico (agua potable), negocios inclusivos, educación y cultura ciudadana.

Una vez definidos los temas centrales, el plan de RSE se somete a revisión en la Facultad de Ciencias de la Administración, el Instituto de Prospectiva, Innovación y Gestión del Conocimiento y el Grupo de Investigación Humanismo y Gestión de la Universidad del Valle. El papel de la universidad en esta actividad es estructurar el plan de acuerdo a los lineamientos del programa Región Responsable. En este ejercicio se formula un proyecto de investigación financiado por Colciencias para caracterizar a la región y a través de herramientas prospectivas de consulta a los actores sociales construir un escenario apuesta para el Norte del Cauca, de acuerdo con la visión de futuro de los actores de la región en función de la pregunta ¿Cómo articular a los diversos actores públicos y privados de la región Norte del Cauca en una estrategia que promueva el desarrollo integral de las empresas, las instituciones, los municipios y las comunidades de los municipios de Caloto, Guachené, Puerto Tejada y Villarica?

Ante esta interrogante, los actores participantes del proceso encuentran que un camino con muchas potencialidades es la Responsabilidad Social Empresarial, que en este contexto muestra su potencial de ser un enfoque articulador y aportar en la construcción de una propuesta particular de desarrollo regional.

\section{Reflexiones y Conclusiones del proceso}

En el proceso de formulación y reflexión que ha permitido construir los cimientos de la Región Socialmente Responsable en el Norte del Cauca ha primado un principio de corresponsabilidad. Aunque la base conceptual del proceso es la responsabilidad social empresarial, esto no implica que se espere una participación exclusiva de las escuelas de administración o de los empresarios que están presentes en la zona. Por el contrario, esta iniciativa ha contado con el concurso de diversos actores, de las fuerzas vivas si se quiere, que le apuestan a un objetivo común con iniciativas articuladas y coherentes.

Uno de los principales aprendizajes de la consolidación de la estrategia ha sido el acercamiento entre actores para la construcción de planes conjuntos de acción. El trabajo independiente al que se tenía costumbre, y la falta de confianza en muchos casos entre actores de la esfera pública y privada, fue el primer punto a superar. En este tema, los procesos de sensibilización y la iniciativa de los actores consolidaron los espacios de acercamiento y diálogo entre el sector empresarial, el gobierno local y las comunidades.

Otro de los aprendizajes que pueden extraerse de la experiencia de la estrategia Región Socialmente Responsable en el Norte del Cauca tiene que ver con el alcance que puede tener una participación activa del sector privado en la esfera del desarrollo regional. La puesta en marcha de acciones que se enmarcan en una gestión de la responsabilidad social, concebida ésta como una reflexión sobre el papel que la empresa juega en la sociedad, pone en evidencia que en desarrollo de su objeto social, la empresa puede realizar acciones a lo largo de su esfera de influencia que superan el cumplimiento de sus objetivos económicos.

Así, la Responsabilidad Social Empresarial deja de ser vista como un tema de filantropía, donaciones, jornadas comunitarias o como problemáticas relacionadas con el mercadeo y el posicionamiento de las marcas, y pasa a ser comprendida como un imperativo en nuestro contexto por las características y demandas sociales sobre el rol de la empresa en la sociedad; asumida como una estrategia integral de negocio responsable de alcance territorial, que fortalece las capacidades de las organizaciones y promueve el desarrollo de la comunidad donde operan, generando oportunidades para la construcción de un espacio de diálogo y articulación entre los diversos actores sociales de una comunidad. En resumen, la RSE puede ser un ejercicio de construcción de confianza.

El desarrollo de una región, entendido como la construcción de capacidades locales y la articulación de los actores en torno a iniciativas comunes impone como desafío el establecimiento de una agenda de trabajo articulado y la construcción de escenarios de desarrollo que 
involucren y beneficien a todos los actores en las diferentes dimensiones de la vida regional. El impulso de parte los empresarios, para una iniciativa de alcance regional como la estrategia Región Socialmente Responsable en el Norte del Cauca, es una evidencia del reconocimiento que empieza a ser cada vez más evidente, sobre la necesidad de incorporar la Responsabilidad Social Empresarial no sólo como un tema de filantropía, donaciones o jornadas comunitarias o como problemáticas relacionadas con la productividad, sino como: 1) un imperativo en nuestro contexto por las características sociales; 2) una estrategia integral de negocio responsable de alcance territorial; 3) una iniciativa que fortalece las capacidades de las organizaciones y promueve el desarrollo de la comunidad donde operan, 4) una oportunidad para la construcción de un espacio de diálogo y articulación entre los diversos actores sociales de una comunidad; 5) un ejercicio de construcción de confianza.

\section{Referencias}

Agulló, X. (2009). Los territorios socialmente responsables. Artículo publicado en suplemento sobre responsabilidad social corporativa del diario mexicano el economista. Recuperado de: www3.eleconomista.com.mx/download. php?file=publicaciones_pdf/especializadas/ responsabilidad/responsabilidad_100309.pdf

Alonso, J. (2006). 10 años de la ley páez. Impacto económico. Universidad icesi.

Alonso, J and Lotero, A. (2008). 10 años de la ley Páez. Transformación de la economía caucana. Universidad Icesi.

Amunorca. (2006). Agenda regional norte caucana para el desarrollo social sostenible 2006-2019.

Banco Interamericano de Desarrollo -BID- (2007). El argumento empresarial de la RSE: 9 casos de américa latina y el caribe.

Betancourt, B; Azuero, A.R; and Martínez, R. (2011). Región Socialmente Responsable Norte del Cauca. Programa editorial Universidad del Valle.

Carroll, A B. (1999). "Corporate Social Responsibility. Evolution of a definitional construct". Business \& society, vol. 38, no. 3, 268-295.
Castillo et al, (2007). "Documento base de los territorios socialmente responsables". Recuperado de internet el 20 de septiembre de 2010 en http://www.mtin.es/uafse/es/cop/ redretos/01.pdf

Davis, K.: (1960). 'Can business afford to ignore corporate social responsibilities?, California Management Review 2, 70-76.

Friedman, M. (1.970). "La responsabilidad social de los negocios es aumentar sus utilidades". En "oficio y arte de la gerencia" Vol II. Págs 195-203.

Fundación Carvajal (2007). Estudio de caracterización social y económico de los trabajadores de Pavco y su entorno inmediato. Guachené - Cauca.

Gardetti, M.A y Muro, A.T. (2009). La base de la pirámide: diferencias entre stuart l. Hart y c. K. Prahalad. En textos sobre la base de la pirámide. Hacia la co-creación de valor y desarrollo. Buenos aires: Dunken.

García, A. (2008). Cultura organizacional y cultura afrocolombiana. Un estudio cualitativo de caso en la planta de Pavco en Guachené - Cauca. Tesis de maestría en administración. Universidad del Valle.

Garriga, E. and Melé, D. (2004). "Corporate Social Responsibility theories: mapping the territory". Journal of business ethics, 53, 1-2, pp 51-71.

Gutiérrez, R; Avella, L; y Villar, R. (2006). Aportes y desafios de la responsabilidad social empresarial en Colombia. Bogotá: Fundación Corona.

Márquez, P; Reficco, E. and Beger, G. (2009). Negocios inclusivos en américa latina. Harvard business review. Mayo.

Porter, M; Kramer, M. (2.002). "The competitive advantage of the corporate philanthropy". The harvard business review. 80(12), 56-69.

Prahalad, C.K. and Hall, S. (2002). The fortune at the bottom of the pyramid. Strategy + business. 26, 54-67

Salinas, L.E. (2010) Región Socialmente Responsable. En: Región Socialmente Responsable 
Norte del Cauca. Colciencias. Instituto de prospectiva, innovación y gestión del conocimiento, universidad del valle. Informe de avance.

Sekn. (2010). Socially inclusive business. Engaging the poor through market initiatives in iberoamerica. David rockefeller center for latin american studies.

Sistema geográfico Agustín Codazzi. (2009). Mapa. división político administrativo departamento del cauca. Recuperado de: http://190.254.22.44/mapas_de_colombia/ igac/mps_politico_admon_dptales/cauca1. pdf

Van Tulder R., and Van der Zwart, A. (2006). "International business-society management linking corporate responsibility and globalisation”. London and New York: routledge.

Wartick and Mahon. (1994): 'Towards a substantive definition of the corporate issue construct: a review and synthesis of literature', business and society 33(3), 293-311.

Visser , W; Matten, D; Pohl, M; and Tolhurst, N. (eds) (2007). The a to $z$ of corporate social responsibility. 